
\title{
3 Research Square \\ Contribution of the Elevated Thrombosis Risk of Males to the Excess Male Mortality Observed in COVID-19: An Observational Study
}

Kenneth R Cohen MD ( $\sim$ ken.cohen@optum.com )

Optum Labs at United Health Group https://orcid.org/0000-0002-6141-9142

David Anderson BSAE

Optum Labs at United Health Group

Sheng Ren, PhD

Optum Labs at United Health Group

David J. Cook MD

Optum Labs at United Health Group

\section{Research Article}

Keywords: Covid-19, Thrombosis, Critical Care

Posted Date: November 22nd, 2021

DOI: https://doi.org/10.21203/rs.3.rs-1097336/v1

License: (a) (i) This work is licensed under a Creative Commons Attribution 4.0 International License.

Read Full License

Version of Record: A version of this preprint was published at BMJ Open on February 1st, 2022. See the published version at https://doi.org/10.1136/bmjopen-2021-051624. 


\section{Abstract}

Background: The mortality rate of COVID-19 is elevated in males compared to females.

Objective: Determine the extent that the elevated thrombotic risk in males relative to females contributes to excess COVID-19 mortality in males.

Design: Observational study.

Setting: Data sourced from electronic medical records from over 200 US hospital systems.

Participants: 60,877 patients hospitalized with COVID-19.

Exposure: Exposure variable: biological sex; key variable of interest: thrombosis.

Main outcome measures: Primary outcome was COVID-19 mortality. We measured: 1) mortality rate of males relative to females, 2) rate of thrombotic diagnoses occurring during hospitalization for COVID-19 in both sexes, and 3) mortality rate when evidence of thrombosis was present.

Results: The COVID-19 mortality rate of males was $29.9 \%$ higher than that of females. Males had a $35.8 \%$ higher rate of receiving a thrombotic diagnosis compared to females. The mortality rate of all patients with a thrombotic diagnosis was $40.0 \%$ - over twice that of COVID-19 patients without a thrombotic diagnosis (adjusted OR 2.50 [2.37 to 2.64], p-value < .001). When defining thrombosis as either a documented thrombotic diagnosis or a D-dimer level $\geq 3.0 \mu \mathrm{g} / \mathrm{mL}, 16.4 \%$ of the excess mortality in male patients could be explained by increased thrombotic risk.

Conclusions and Relevance: Our findings suggest the higher COVID-19 mortality rate in males may be significantly accounted for by the elevated risk for thrombosis among males. Understanding the mechanisms that underlie increased male thrombotic risk may allow for the advancement of effective anticoagulation strategies that reduce COVID-19 mortality in males.

\section{Strengths And Limitations Of The Study}

- Data derived from over 52,000 hospitalized patients with COVID-19 comprised of blended claims and EHR data

- NLP engine allowing for detailed interrogation of EHR data

- Independent manual verification of NLP engine accuracy

- Data elements could be missed if not expressed in a standard fashion that can be recognized by the NLP engine

- True magnitude of underlying thrombosis in this population could be underestimated by both claims analysis and NLP interrogation of EHR data 


\section{Introduction}

Male sex has emerged as a risk factor for increased COVID-19 morbidity and mortality, with the risk of mortality in males being $30 \%-40 \%$ higher than in females. ${ }^{1,2}$ This heightened mortality risk for males is independent of advancing age, and cannot be fully explained by higher rates among males of other comorbidities known to increase mortality in COVID-19, such as obesity, diabetes mellitus, hypertension, or underlying cardiopulmonary disease. ${ }^{3,4}$ Therefore, the etiology of this sex difference in COVID-19 mortality is largely unexplained.

Thrombosis appears to play an important role in the morbidity and mortality of COVID-19, with patients at increased risk of both micro- and macrovascular thrombosis. In a meta-analysis of 42 studies representing data from 8,271 COVID-19 patients, the authors documented an overall venous thromboembolism rate of $21 \%$ and a pulmonary embolism rate of $13 \%$. For patients admitted to the ICU, the rate of thromboembolism was even higher, at $31 \%$. Notably, the pooled odds of mortality were $74 \%$ higher among COVID-19 patients who developed thromboembolism, compared to those who did not. ${ }^{5}$

Additionally, thromboses may go undiagnosed during a hospital stay for COVID-19. Markedly elevated Ddimer levels are highly correlated with underlying thrombosis, confirmed in two recent studies. In the first study, a level of $3.0 \mu \mathrm{g} / \mathrm{mL}$ had a sensitivity of $70.0 \%$, specificity of $96.7 \%$ with a positive predictive value of $87.5 \%$ for underlying thrombosis. ${ }^{6}$ In the second study, a level $>2.5 \mathrm{ug} / \mathrm{mL}$ had a sensitivity of $63 \%$ and a specificity of $85 \%$ for the diagnosis of pulmonary embolism. ${ }^{7}$

The elevated thrombotic risk of males relative to females was well-established in the medical literature long before the emergence of COVID-19. In multiple studies of unprovoked deep venous thrombosis and pulmonary embolus (DVT/PE) where hereditary thrombophilia was excluded, the rate of recurrent thrombosis in males has been reported to be anywhere from two to more than three times higher than females. ${ }^{8-12}$ Despite extensive research on the topic, the etiology of this sex-based difference in the thrombotic risk of males remains unknown. ${ }^{13}$

We therefore understand that males are at risk for higher COVID-19 mortality, that COVID-19 mortality risk is increased when patients have underlying thrombosis, and that unrelated to COVID-19, males are more prone to thrombosis relative to females. It is therefore plausible that the elevated thrombotic risk of males relative to females may partially explain the excess mortality rate observed in males with COVID-19. In this study we sought to understand whether males with COVID-19 have a higher rate of thrombosis compared to females with COVID-19, and whether a higher rate of thrombosis contributes to the excess mortality seen in males.

\section{Methods}

\section{Data Source}


To explore a potential connection between thrombosis, sex, and mortality risk, we analyzed hospitalization data from over 200 geographically dispersed hospital systems. From this data we selected patients who were hospitalized for COVID-19 between March 3, 2020, and June 11, 2021. There were no exclusions for other comorbidities or underlying diseases. We limited our analysis to those patients who survived and were discharged from the hospital, those discharged to hospice care, those who died in the hospital, and those who died after being discharged to home. Patients who died soon after discharge to home or who were discharged to hospice care were treated as deceased cases due to COVID-19. COVID-19 readmission cases were excluded from our study.

The data for this study was sourced from EMR data and post-EMR coding. These EMRs were processed with a natural language processing (NLP) engine that produces a homogenized set of codified and noncodified information, including lab results, medications, symptoms, and various observational extracts of text from the EMR. The at-scale extraction of this detailed information allows insight into the clinical manifestations of the COVID-19 population. We examined the subsets of patients who survived COVID19 compared to those who expired. For each of the groups, we compared the male and female incidence of receiving a thrombosis diagnosis code while hospitalized for COVID-19.

\section{Outcomes and Study Variables}

The primary outcome of interest was mortality from COVID-19. The exposure variable was biological sex, and the key variable of interest was thrombosis. We classified thrombotic diagnosis codes into four major conditions: (1) myocardial infarction (MI), (2) DVT/PE, (3) stroke, and (4) peripheral artery occlusion (PAO). A full list of ICD-10 codes used to identify thrombosis can be found in Supplementary Table e1.

Because thrombotic diagnoses are underreported in inpatients with COVID-19, $1{ }^{14}$ we also examined the peak D-dimer value of the subset of hospitalized patients in whom this was measured. A D-dimer level $\geq$ $3.0 \mu \mathrm{g} / \mathrm{mL}$ was used as a surrogate for underlying thrombosis when a thrombotic diagnosis code was absent.

\section{Statistical Methods}

To determine whether the elevated thrombotic risk in males is associated with higher COVID-19 mortality, we used mediation analysis, ${ }^{15}$ where elevated thrombotic risk was the mediator between the outcome (mortality) and the exposure variable (gender). Mediation analysis consists of three steps, which are illustrated in Figure 1. First, we needed to confirm that male patients had higher mortality rate than female patients in our study population. Second, we needed to confirm that males had a higher incidence of a thrombosis diagnosis relative to females. Third, we needed to demonstrate that both males and females with thrombosis diagnoses had a higher mortality rate than those who did not. If all three of these conditions are supported by statistically significant differences between groups, we can combine regression models from the first and third hypothesis to estimate the proportion of sex effect on mortality that can be explained by the elevated thrombotic risk. Besides estimating this proportion, we also 
examined the effect of a thrombosis diagnosis together with evidence of a D-dimer level $\geq 3.0 \mu \mathrm{g} / \mathrm{mL}$, recognizing that such levels are also likely indicative of underlying thrombosis.

Logistic regression models were used in each of the three steps. The response variables and covariates of interest are illustrated in Figure 1. We used the Wald test for regression coefficients in logistic regression models to assess statistical significance in all steps. All hypothesis tests were one-sided at the 0.05 significance level. We report $95 \%$ confidence intervals and one-tailed p-values. We choose the more powerful one-sided test because all three associations described in Figure 1/Table 2 (male and higher risk of mortality, male and higher risk of thrombosis, and thrombosis and higher risk of mortality) are well documented in literature as we discussed in the Introduction section, and we only wanted to test if these hypotheses were true with respect to directions supported by literatures. All regression models were adjusted for age (binary variable of age $>65$ or not) and co-morbidities derived from the Charlson Index. ${ }^{16}$ These co-morbidities can be found in Supplementary Material Table e2. Note that two comorbidities - myocardial infarction and cerebrovascular diseases are not adjusted in our regression models since they overlap with our thrombosis definitions shown in Supplementary Material Table e1.

The proportion of excess male mortality explained by the elevated thrombotic risk is defined by the ratio of the mediation effect to the total effect (mediation + direct effect) of gender on mortality. This proportion was estimated using the methods explained in this paper, ${ }^{17}$ which was implemented by an $\mathrm{R}$ package "mediation". ${ }^{18}$ Our R codes for mediation analysis using this package can be found in Supplementary Materials III. Because thrombotic diagnoses are underreported in inpatients as discussed above, we estimated the proportion based on the presence of a thrombosis diagnoses only as well as a definition of elevated thrombotic risk that included D-dimer values $>3.0 \mu \mathrm{g} / \mathrm{mL}^{19}$ or presence of a thrombotic diagnosis. We also reported the proportion explained by thrombosis diagnoses only on the subset whose D-dimer was measured to make sure the proportion explained by elevated thrombotic risk was not subject to selection bias. The upper bound of the D-dimer normal range $(0.5 \mu \mathrm{g} / \mathrm{mL})$ was used to normalize D-dimer values.

\section{Patient and Public Involvement}

As this study represents an exploratory secondary analysis of existing, de-identified patient data, no IRB review and approval were required, and no patients or members of the public were involved in the design, conduct, reporting, or dissemination plans of our research.

\section{Results}

Our study population was comprised of 60,877 COVID-19 patients with an average age of 62 years. There were slightly more male patients (51.3\%) than female patients. Among study patients, $16.2 \%$ had a reported thrombotic diagnosis during their hospital stay and $20.4 \%$ died or were discharged to hospice care. We also identified a subset of 31,817 patients (52.3\%) out of the study population (with or without a thrombotic diagnosis) who had D-dimer values measured during their hospital stay. Normalized mean 
peak D-dimer during a hospital stay were 4.35 (435\% of the normal range upper bound). Characteristics of the full study population and this patient subset are shown in Table 1. Percentages of comorbid conditions stratified by gender and thrombosis diagnosis are shown in Supplementary Tables e2 and e3 for these two populations.

\section{Table 1. Characteristics of the Full Hospitalized Population and the D-dimer Subset}

\begin{tabular}{|lll|}
\hline Study sample characteristics & \multicolumn{2}{l|}{ Summary statistics $^{\mathrm{a}}$} \\
\cline { 2 - 3 } & Full population dataset & D-dimer analysis subset \\
\hline Sample Size & 60,877 & 31,817 \\
\hline Deceased & $12,430(20.42)$ & $6,633(20.85)$ \\
\hline Thrombophilia & $9,845(16.17)$ & $5,160(16.22)$ \\
\hline Thrombosis history & $4,085(6.71)$ & $2,147(6.75)$ \\
\hline Mechanical ventilation & $6,803(11.17)$ & $3,941(12.39)$ \\
\hline Deceased or intubated & $13,960(22.93)$ & $7,478(23.50)$ \\
\hline MI & $3,625(5.96)$ & $1,732(5.44)$ \\
\hline DVT/PE & $5,150(8.46)$ & $3,000(9.43)$ \\
\hline Stroke & $1,891(3.11)$ & $854(2.68)$ \\
\hline PAO & $290(0.48)$ & $100(0.31)$ \\
\hline Age average (standard deviation) & $61.70(18.20)$ & $62.84(16.54)$ \\
\hline Sex: male & $31,197(51.25)$ & $16,969(53.33)$ \\
\hline D-dimer average & - & $435.39 \%$ \\
\hline D-dimer median & - & $184.40 \%$ \\
\hline
\end{tabular}

Abbreviations: MI, myocardial infarction; DVT, deep vein thrombosis; PAO, peripheral arterial occlusion.

${ }^{a}$ With the exception of D-dimer statistics, sample size, and age, data are otherwise reported as number (percentage)

of patients within each dataset. D-dimer statistics are based on the upper bound of the normal range for each measure.

We found that the mortality rate in males with COVID-19 was higher by $29.9 \%$ compared to females with COVID-19 (an absolute rate difference of 5.3\%; adjusted odds ratio (OR) = 1.41 [1.35 to 1.48], $p<$ .001). Compared to females with COVID-19, males with COVID-19 had a rate of receiving a thrombotic diagnosis during their hospital stay that was $35.8 \%$ higher (an absolute difference of $4.9 \%, \mathrm{OR}=$ 
1.34 [1.28 to 1.40$], p<.001)$, confirming the higher rate of thrombotic diagnoses in males. Additionally, we found an over two-fold difference in mortality between patients with and without a thrombotic diagnosis ( $40.0 \%$ vs. $16.7 \%$; adjusted $O R=2.50$ [2.37 to 2.64], $p<.001)$. Having verified that thrombosis is more prevalent in males $(\mathrm{OR}=1.34)$ and that thrombosis is a strong risk factor for mortality $(\mathrm{OR}=$ 2.50), we conclude that the elevated thrombotic risk accounts for a portion of the excess mortality in males. All results of this three-steps mediation analysis are shown in Table 2.

\section{Table 2. Full Mediation Analysis Results of Thrombosis Diagnosis}


Mediation analysis steps

\section{Step 1:}

Documentation of the higher mortality rate in males
Step 2:

Documentation of the higher rate of thrombosis in males

\begin{tabular}{lll} 
& Female & Male \\
\hline Number of patients & 29,680 & 31,197 \\
\hline Survived & 24,438 & 24,009 \\
\hline Deceased & 5,242 & 7,188 \\
\hline $\begin{array}{l}\text { Sex distribution among } \\
\text { deceased patients }\end{array}$ & $42.2 \%$ & $57.8 \%$ \\
\hline $\begin{array}{l}\text { Rate of deceased patients } \\
\text { by sex }\end{array}$ & $17.7 \%$ & $23.0 \%$ \\
\hline $\begin{array}{l}\text { Unadjusted odds ratio } \\
\text { Adjusted odds ratio }\end{array}$ & $\begin{array}{l}1.40[1.34,1.45] \text { p-value } \\
<.001\end{array}$ \\
\hline $\begin{array}{l}1.41[1.35,1.48] \text { p-value } \\
<.001\end{array}$
\end{tabular}

\begin{tabular}{lll} 
& Female & Male \\
\hline Number of patients & 29,680 & 31,197 \\
\hline No thrombosis diagnosis & 25,625 & 25,407 \\
\hline $\begin{array}{l}\text { Had thrombosis } \\
\text { diagnosis }\end{array}$ & 4,055 & 5,790 \\
\hline $\begin{array}{l}\text { Sex distribution among } \\
\text { thrombosis patients }\end{array}$ & $41.2 \%$ & $58.8 \%$ \\
\hline $\begin{array}{l}\text { Thrombosis prevalence } \\
\text { by sex }\end{array}$ & $13.7 \%$ & $18.6 \%$ \\
\hline $\begin{array}{l}\text { Unadjusted odds ratio } \\
\text { Adjusted odds ratio }\end{array}$ & $\begin{array}{l}1.44[1.38,1.50] \text { p-value } \\
\end{array}$ & $\begin{array}{l}1.34[1.28,1.40] \\
<.001\end{array}$ \\
\hline
\end{tabular}

\section{Step 3:}

Documentation of the higher mortality rate associated with a thrombosis diagnosis

\begin{tabular}{lll} 
& $\begin{array}{l}\text { No } \\
\text { thrombosis }\end{array}$ & $\begin{array}{l}\text { Had } \\
\text { thrombosis }\end{array}$ \\
\hline Number of patients & 51,032 & 9,845 \\
\hline Survived & 42,537 & 5,910 \\
\hline Deceased & 8,495 & 3,935 \\
\hline $\begin{array}{l}\text { Thrombosis distribution } \\
\text { among deceased patients }\end{array}$ & $68.3 \%$ & $31.7 \%$ \\
\hline $\begin{array}{l}\text { Mortality rates by } \\
\text { thrombosis }\end{array}$ & $16.7 \%$ & $40.0 \%$ \\
\hline
\end{tabular}


Unadjusted odds ratio

Adjusted odds ratio

Adjusted odds ratio for

sex
3.33 [3.18, 3.49] p-value

$<.001$

$2.50[2.37,2.64]$ p-value

$<.001$

$1.38[1.31,1.44]$ p-value

$<.001$

Based on these findings, we then sought to determine what proportion of the excess male mortality in COVID-19 patients might be related to the elevated thrombotic risk. When using only a documented diagnosis of thrombosis, the proportion of the mortality effect explained by thrombosis was significant at $12.1 \%$ ( $p$.001), with a 95\% confidence interval [9.44\%, 15.3\%] (Table 3). Because of underreporting of thrombotic diagnoses in hospitalized patients with COVID-19, we also considered a D-dimer level $\geq 3.0$ $\mu \mathrm{g} / \mathrm{mL}$ as a surrogate of underlying thrombosis. We therefore combined the proportion of increased mortality in males as predicted by a thrombotic diagnosis with the proportion explained by a D-dimer level $>3.0 \mathrm{ug} / \mathrm{ml}$. In doing so, the total proportion of excess male mortality potentially explained by the elevated thrombosis risk in males increased to $16.4 \%$ with a $95 \%$ confidence interval [11.4\%, 22.6\%] when the thrombosis definition was expanded to include D-dimer levels $>3.0 \mu \mathrm{g} / \mathrm{mL}$. (Table 3 ). Note that in this subset of patients who had D-dimer measured (52.3\% of the study cohort), the proportion of COVID-19 cases with a documented thrombotic diagnosis alone was $10.6 \%$ (Table 3 ), which was very close to the $12.1 \%$ of cases with a documented thrombotic diagnosis in the study cohort. This indicates that an additional $5.8 \%$ of cases can be explained by elevated D-dimer levels, and that there was almost no bias in the selection of the sub-cohort that had D-dimer measured.

\section{Table 3. Proportion of Excess Mortality Explained by Elevated Thrombotic Risk}

\begin{tabular}{|ll|}
\hline Mediators & Proportion mediated (Explained) \\
\hline Thrombosis diagnosis on whole dataset & $12.1 \%[9.44 \%, 15.3 \%] \mathrm{p}$-value $<.001$ \\
\hline Thrombosis diagnosis on D-dimer subset & $10.6 \%[7.01 \%, 15.2 \%] \mathrm{p}$-value $<.001$ \\
\hline Thrombosis diagnosis codes or D-dimer $>3.0 \mu \mathrm{g} / \mathrm{mL}$ & $16.4 \%[11.4 \%, 22.6 \%] \mathrm{p}$-value $<.001$ \\
\hline
\end{tabular}

\section{Discussion}

The data extracted from our large population of hospitalized patients with COVID-19 confirms a higher mortality rate among those patients with thrombosis. It also demonstrates that the incidence of thrombosis is higher in males relative to females and may explain up to $16.4 \%$ of the excess mortality seen in males with COVID-19.

Because the known genetic risks for thrombophilia (factor V Leiden, prothrombin G20210A, protein C and $S$ abnormalities, etc.) are not sex-linked chromosomal mutations, they cannot explain this excess male 
risk. There are, however, genetic mutations found on the $\mathrm{X}$ and $\mathrm{Y}$ chromosomes which might explain a portion of this increased thrombophilia in males. ${ }^{12}$ Additionally, severe COVID-19 infection is itself associated with a prothrombotic state. Although the prothrombotic mechanisms are not fully understood, several have been postulated. Activation of the coagulation cascade through direct viral invasion of the vascular endothelium may cause severe endothelial injury with disruption of fibrinolytic activity and release of von-Willebrand factor. ${ }^{20}$ Thrombosis may be triggered by the generalized cytokine activation seen in severe COVID-19 with resultant activation of platelets and the complement system. ${ }^{21}$ Lastly, COVID-19 has been associated with the development of procoagulant autoantibodies, including antiphospholipid antibodies. ${ }^{22}$ Diabetes has also been shown to independently increase the risk of thrombosis in hospitalized patients with COVID-19. ${ }^{23}$

To our knowledge, this is the first study to specifically address the significantly elevated risk of thrombosis and its associated excess mortality in males with COVID-19 relative to females. Although mortality as a function of sex was not specifically addressed in the meta-analysis of the 42 studies noted in the introduction, ${ }^{5}$ that study also documented that thrombosis contributes to excess mortality in COVID-19. Moreover, among those 42 studies, 29 documented the sex of the patients. Of those 29 studies, 27 (93\%) documented a higher percentage of thromboembolism in males relative to females. Of all cases of thromboembolism across those 29 studies, $70 \%$ occurred in males and $30 \%$ occurred in females.

Studies of thromboprophylaxis among hospitalized COVID-19 patients have documented improved outcomes. A systematic review and pooled analysis of 35 studies looked at anticoagulation strategies in 4,685 hospitalized patients with COVID-19. ${ }^{24}$ This review suggested that standard prophylactic doses of anticoagulation were associated with significant reductions in venous thromboembolism and arterial thrombosis events, with intermediate and therapeutic doses of anticoagulation providing no additional benefit. A more recent RCT documented improved outcomes with full dose anticoagulation compared with thromboprophylaxis in a noncritically ill population hospitalized with COVID-19. The benefit was most apparent in the subset of patients with the highest D-dimer levels. ${ }^{25} \mathrm{~A}$ second RCT found that compared to standard heparin thromboprophylaxis, therapeutic doses of low molecular weight heparin reduced major thromboembolism and death in a population of high risk hospitalized patients with COVID19 and very elevated D-dimer levels. ${ }^{26}$ These studies did not report stratification of the anticoagulation benefit by sex of the patients.

Our study has limitations. NLP extraction of EMR data may miss certain data elements if they are not expressed in a standard fashion that can be recognized by the NLP engine. The accuracy of the output of the NLP engine was confirmed in a subset of patients by manual chart review. Similarly, analysis of claims data might miss patients with underlying thrombotic diagnoses if these diagnoses were assumed to be part of the COVID-19 clinical syndrome and therefore not independently submitted. Lastly, when interpreting the results from the D-dimer analysis it cannot be stated with certainty that a markedly elevated D-dimer level is related to underlying thrombosis. 
In conclusion, our data support the concept that a significant portion of the excess male mortality in COVID-19 is related to the elevated risk of thrombosis in males relative to females. Understanding the mechanisms that drive the elevated thrombotic risk in males, as well as those that drive thrombosis in COVID-19, may allow for the development of more effective anticoagulation strategies that reduce the mortality risk for males diagnosed with COVID-19. To help develop these strategies, re-analysis of outcome by sex in recently published trials and new randomized clinical trials are suggested to examine whether more intense anticoagulation regimens in males hospitalized with COVID-19 will reduce thromboses and improve clinical outcomes including mortality.

\section{Declarations}

\section{ACKNOWLEDGEMENTS}

Author Contributions: Dr. Ken Cohen, Mr. Dave Anderson, Dr. Sheng Ren, and Dr. David Cook had full access to all of the data in the study and take responsibility for the integrity of the data and the accuracy of the data analysis.

Concept and design: All authors.

Acquisition, analysis, or interpretation of data: All authors.

Drafting of the manuscript: All authors.

Critical revision of the manuscript for important intellectual content: All authors and Amy Okaya, MPH, MBA, OptumLabs.

Editing of manuscript: Amy Okaya.

Statistical analysis: Sheng Ren.

Supervision: Ken Cohen.

Competing interests: All authors have completed the ICMJE uniform disclosure form at http://icmje.org/disclosure-of-interest/ and declare: all authors had financial support from UnitedHealth Group for the submitted work; no financial relationships with any organizations that might have an interest in the submitted work in the previous three years; no other relationships or activities that could appear to have influenced the submitted work.

Funding: The study was funded by OptumLabs, the research and development arm of UnitedHealth Group, and the authors Drs. Cohen, Cook, Ren, and Mr. Anderson are full-time employees of UnitedHealth Group. These authors played an active role in all aspects of study development, including the design and conduct of the study; collection, management, analysis, and interpretation of the data; preparation, review, or approval of the manuscript; and decision to submit the manuscript for publication. 
Ethics approval: As this study represents an exploratory secondary analysis of existing, de-identified patient data, no IRB review and approval was required.

IRB Tracking number 2021-0063. IRB waiver granted

United Health Group IRB

Federalwide Assurance \#: FWA00028881

OHRP Registration \#: IORG0010356

Transparency statement: The lead author, Dr. Ken Cohen, affirms that this manuscript is an honest, accurate, and transparent account of the study being reported; that no important aspects of the study have been omitted; and that any discrepancies from the study as planned (and, if relevant, registered) have been explained.

Data availability statement: The data are not available for public use but, under certain conditions, may be made available to editors and their approved reviewers under a data-use agreement to confirm the findings of the current study.

\section{References}

1. Martins-Filho PR, Souza Tavares CS, Santos VS. Factors associated with mortality in patients with COVID-19: A quantitative evidence synthesis of clinical and laboratory data. Eur J Intern Med. 2020:76:97-99. DOI: 10.1016/j.ejim.2020.04.043

2. The Sex, Gender, and COVID-19 Project [Internet]. Men, sex, gender and COVID-19. [Cited December 28, 2020]. Available from: https://globalhealth5050.org/the-sex-sex-and-COVID-19-project/men-sexsex-and-COVID-19/

3. Docherty AB, Harrison EM, Green CA, et al. Features of 20133 UK patients in hospital with covid-19 using the ISARIC WHO Clinical Characterisation Protocol: prospective observational cohort study. BMJ. 2020;369:m1985. DOI:10.1136/bmj.m1985

4. Peckham $\mathrm{H}$, de Gruijter NM, Raine $\mathrm{C}$, et al. Male sex identified by global COVID-19 meta-analysis as a risk factor for death and ITU admission. Nat Commun. 2020;11(1):6317. DOI:10.1038/s41467-02019741-6

5. Malas MB, Naazie IN, Elsayed N, Mathlouthi A, Marmor R, Clary B. Thromboembolism risk of COVID19 is high and associated with a higher risk of mortality: A systematic review and meta-analysis.

EClinicalMedicine [Intenet]. 2020 Nov. Available from https://pubmed.ncbi.nlm.nih.gov/33251499/ DOI:10.1016/j.eclinm.2020.100639

6. Cui S, Chen S, Li X, Liu S, Wang F. Prevalence of venous thromboembolism in patients with severe novel coronavirus pneumonia. J Thromb Haemost. 2020;18(6):1421-1424. DOI:10.1111/jth.14830 
7. Logothetis CN, Weppelmann TA, Jordan A, Hanna C, Zhang S, Charkowick S, et al. D-Dimer Testing for the Exclusion of Pulmonary Embolism Among Hospitalized Patients With COVID-19. JAMA Netw Open. 2021 Oct 8;4(10):e2128802. DOI:10.1001/jamanetworkopen.2021.28802

8. Christiansen SC, Lijfering WM, Helmerhorst FM, Rosendaal FR, Cannegieter SC. Sex difference in risk of recurrent venous thrombosis and the risk profile for a second event. J Thromb Haemost. 2010; 8(10):2159-68. DOI: 10.1111/j.1538-7836.2010.03994.x

9. Baglin T, Luddington R, Brown K, Baglin C. High risk of recurrent venous thromboembolism in men. $J$ Thromb Haemost. 2004;2(12):2152-5. DOI: 10.1111/j.1538-7836.2004.01050.x

10. Rodger MA, Kahn SR, Wells PS, et al. Identifying unprovoked thromboembolism patients at low risk for recurrence who can discontinue anticoagulant therapy. CMAJ. 2008;179(5):417-26. DOI: 10.1503/cmaj.080493

11. McRae S, Tran H, Schulman S, Ginsberg J, Kearon C. Effect of patient's sex on risk of recurrent venous thromboembolism: A meta-analysis. Lancet. 2006;368(9533):371-8. DOI: 10.1016/S01406736(06)69110-1

12. Douketis J, Tosetto A, Marcucci M, et al. Risk of recurrence after venous thromboembolism in men and women: Patient level meta-analysis. BMJ. 2011;342:d813. DOI: 10.1136/bmj.d813

13. Roach RE, Cannegieter SC, Lijfering WM. Differential risks in men and women for first and recurrent venous thrombosis: the role of genes and environment. $J$ Thromb Haemost. 2014 Oct;12(10):15931600. DOI: $10.1111 /$ jth. 12678.

14. Piazza G, Morrow DA. Diagnosis, Management, and Pathophysiology of Arterial and Venous Thrombosis in COVID-19. JAMA. 2020;324(24):2548-2549. DOI:10.1001/jama.2020.23422

15. Baron RM, Kenny DA. The moderator-mediator variable distinction in social psychological research: conceptual, strategic, and statistical considerations. J Pers Soc Psychol. 1986;51(6):1173-1182. DOI:10.1037//0022-3514.51.6.1173

16. Quan H, Sundararajan V, Halfon P, Fong A, Burnand B, Luthi JC, et al. Coding algorithms for defining comorbidities in ICD-9-CM and ICD-10 administrative data. Med Care. 2005; 43(11):1130-1139. DOI: 10.1097/01.mlr.0000182534.19832.83

17. Imai, K., Keele, L., \& Tingley, D. (2010). A general approach to causal mediation analysis. Psychological methods, 15(4), 309.

18. Tingley D, Yamamoto T, Hirose K, Keele L, Imai K. mediation: R Package for causal mediation analysis. J Stat Soft [Internet]. 2014;59(1):1-38. DOI:10.18637/jss.v059.i05. (accessed Nov 2020)

19. Zhang L, Yan X, Fan Q, et al. D-dimer levels on admission to predict in-hospital mortality in patients with Covid-19. J Thromb Haemost. 2020;18(6):1324-1329. DOI:10.1111/jth.14859

20. Ackermann M, Verleden SE, Kuehnel M, Haverich A, Welte T, Laenger F, et al. Pulmonary Vascular Endothelialitis, Thrombosis, and Angiogenesis in Covid-19. NEJM. 2020 Jul 9;383(2):120-8. DOI: 10.1056/NEJMoa2015432

21. Magro C, Mulvey JJ, Berlin D, Nuovo G, Salvatore S, Harp J, et al. Complement associated microvascular injury and thrombosis in the pathogenesis of severe COVID-19 infection: A report of 
five cases. Trans/ Res. 2020 Jun 1;220:1-13. DOl:10.1016/j.trsl.2020.04.007

22. Piazza G, Morrow DA. Diagnosis, Management, and Pathophysiology of Arterial and Venous Thrombosis in COVID-19. JAMA. 2020;324(24):2548-2549. DOI:10.1001/jama.2020.23422

23. Calvisi SL, Ramirez GA, Scavini M, Da Prat V, Di Lucca G, Laurenzi A, et al. Thromboembolism risk among patients with diabetes/stress hyperglycemia and COVID-19. Metabolism. 2021 Oct;123:154845.DOI: 10.1016/j.metabol.2021.154845

24. Patell R, Chiasakul T, Bauer E, Zwicker JI. Pharmacologic Thromboprophylaxis and Thrombosis in Hospitalized Patients with COVID-19: A Pooled Analysis. Thromb Haemost. 2021 Jan;121(1):76-85.

25. ATTACC Investigators, ACTIV-4a Investigators, REMAP-CAP Investigators, et al. Therapeutic Anticoagulation with Heparin in Noncritically III Patients with Covid-19. N Engl J Med. 2021;385(9):790-802. DOI:10.1056/NEJMoa2105911

26. Spyropoulos AC, Goldin M, Giannis D, Diab W, Wang J, Khanijo S, et al. Efficacy and Safety of Therapeutic-Dose Heparin vs Standard Prophylactic or Intermediate-Dose Heparins for Thromboprophylaxis in High-risk Hospitalized Patients With COVID-19: The HEP-COVID Randomized Clinical Trial. JAMA Intern Med. Published online 2021 Oct 7; doi.org/10.1001/jamainternmed.2021.6203 (accessed 21 Oct 2021).

\section{Figures}

\section{Mediation Analysis Flowchart}

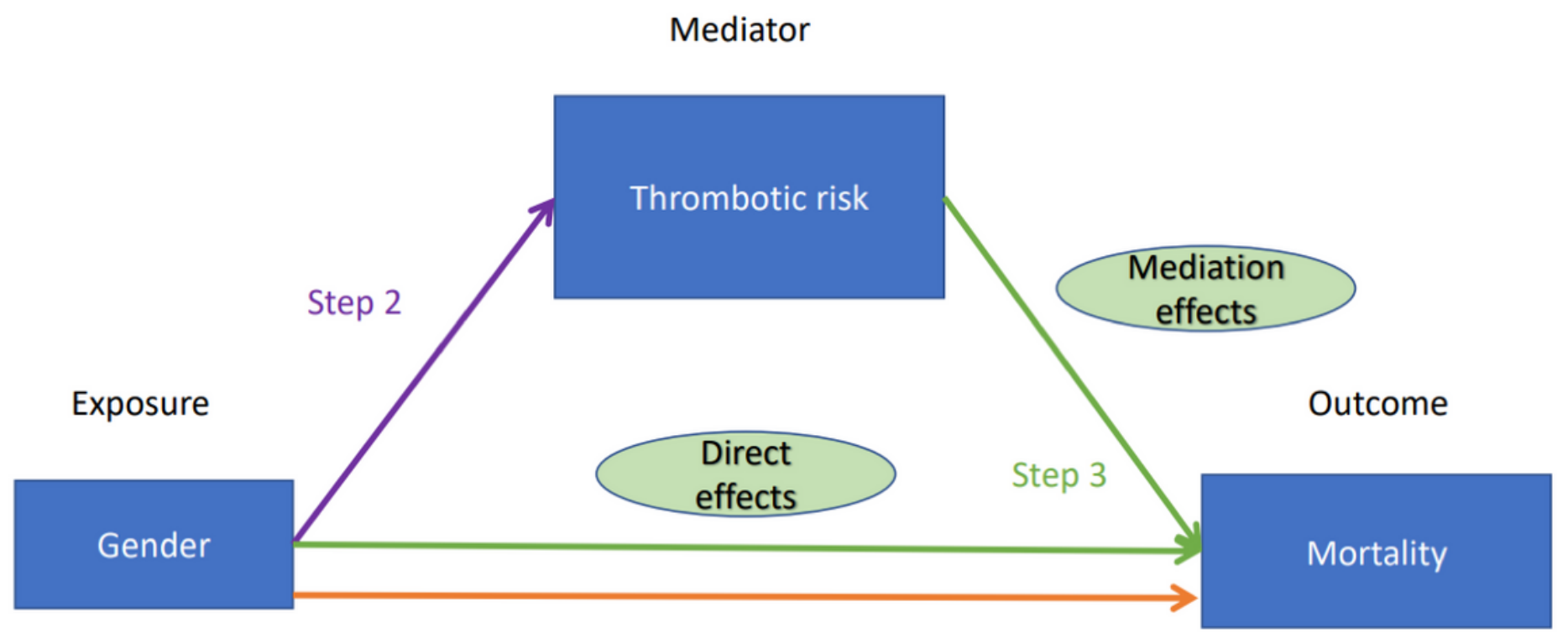

Step 1

Each arrow (step) is a regression model. It starts from a covariate of interest and points to the response variable.

Figure 1 
Mediation Analysis Flowchart

\section{Supplementary Files}

This is a list of supplementary files associated with this preprint. Click to download.

- MaleMortSupplementaryMaterialFinal.docx 